\title{
Hyperspectral evaluation of skin blood oxygen saturation at baseline and during arterial occlusion
}

Z. Marcinkevics, U. Rubins, A. Grabovskis, J. Cimurs, A. Caica

Z. Marcinkevics, U. Rubins, A. Grabovskis, J. Cimurs, A. Caica,

"Hyperspectral evaluation of skin blood oxygen saturation at baseline and during arterial occlusion," Proc. SPIE 10685, Biophotonics: Photonic Solutions for Better Health Care VI, 106851A (17 May 2018); doi: 10.1117/12.2306131

SPIE. Event: SPIE Photonics Europe, 2018, Strasbourg, France 


\title{
Hyperspectral evaluation of skin blood oxygen saturation at baseline and during arterial occlusion
}

\author{
Z. Marcinkevics*a, U. Rubins ${ }^{\mathrm{b}}$, A. Grabovskis ${ }^{\mathrm{b}}$, J. Cimurs $^{\mathrm{b}}$, A. Caica ${ }^{\mathrm{a}}$ \\ ${ }^{a}$ Department of Human and Animal Physiology, University of Latvia, Jelgavas str.1, Riga, LV-1586, \\ Latvia; \\ ${ }^{\mathrm{b}}$ Biophotonics Laboratory of Institute of Atomic Physics and Spectroscopy, University of Latvia, \\ Raina Blvd.19, Riga, LV-1586, Latvia;
}

\begin{abstract}
Skin capillary blood oxygen saturation is a clinically important diagnostic parameter, which provides valuable information for timely treatment of pathological conditions e.g. sepsis, hypoxemia or decompression illness. Hyperspectral imaging is non-invasive optical techniques with high clinical potential, however its use for skin blood oxygen saturation detection is still challenging, therefore in the present study, a method for in-vivo manipulation of skin oxygen saturation was developed, and reliability of the method evaluated by means of hyperspectral imaging in detection of oxygen saturation. In order to produce alterations of skin capillary blood parameters and oxygen saturation, the proximal phalanx of the right middle finger was occluded with a pneumatic cuff for 25 minutes. During the last minute of occlusion, the hyperspectral cubes (HIS) of both occluded and intact finger were captured, and capillary blood sample was collected for analysis with portable whole blood analyzer (REF). The group mean values for $\mathrm{SaO}_{2}$ in intact finger skin was HIS: $89.46 \% \pm 8.79 \%$ versus REF: $95.13 \pm 1.46 \%$ and in occluded finger HSI: $25.85 \% \pm 14.00 \%$, versus REF: $22.73 \pm 9.09 \%$ displaying a small difference between two independent techniques, which indicate the reliability of finger occlusion model.
\end{abstract}

Keywords: Hyperspectral imaging, oxygen saturation, skin microcirculation, arterial occlusion, tunable filters.

\section{INTRODUCTION}

Blood oxygen saturation is a clinically important diagnostic parameter, which provides valuable information for timely treatment of several pathological conditions e.g. sepsis, hypoxemia or decompression illness.

Traditional monitoring of saturation is performed in arterial blood as it provides transport of oxygen to the tissue. The gold standard for such non-invasive saturation detection is well known pulse oximetry technique- first proposed in the early 1930s. This method is easy to use and reliable in most ambulatory settings ${ }^{1}$. However it sometimes fails in emergency situation when monitoring critically ill patients. Another alternative is transcutaneous gas analysis in the tissue using Severinghaus and Clark type electrodes which provide information comparative to arterial blood ${ }^{2}$. This method is widely used in neonates monitoring because of its noninvasiveness, hence its reliability largely depends on tissue perfusion level, and in case of restricted or impaired skin microcirculation, $\mathrm{pO}_{2}$ value tends to be underestimated and $\mathrm{pCO}_{2}$ overestimated.

For this reason many invasive techniques were developed and presently available to clinicians. Most common operation principles are either use of lab-on-chip technology and at bedside in addition to saturation, measure blood gases, $\mathrm{pH}$ levels and electrolytes such as (iSTAT, Abbott) or utilize photo spectrometric approach and measure concentration of oxy and deoxy hemoglobin in the whole blood using absorption spectra (Avoximeter, Accriva Diagnostics). All of them have several inherent drawbacks- invasiveness with consequent risk of infections, and requirement of a relatively large blood sample, which is not an obstacle during arterial or venous blood measurement (sampling from the blood vessel with the syringe) but substantially limit application of methods in analysis of capillary blood from the skin. Moreover, all aforementioned techniques fail in providing local variation of blood saturation in the skin. Which is crucially important for assessment of cutaneous blood saturation in pathologies such as Raynaud's disease and septic mottling - 
discoloration and purple patches on the skin over the knee, where heterogeneity of saturation play an important diagnostic role.

Recently, non-invasive contactless optical techniques are gaining acceptance in clinics as they provide information at bed-side without discomfort, risk of infection and requirements for expensive disposable supplies utilized in invasive procedures.

Hyperspectral imaging is among them and has already proven its clinical potential in detection of skin malformations ${ }^{3}$, however its use for skin blood oxygen saturation detection is still challenging because of structural non-uniformity of skin and complex cutaneous blood circulation ${ }^{4}$. Traditionally, development and evaluation of such methods require tissue phantom measurements; however even advanced phantoms still barely mimic the real skin with its complex microvasculature. Therefore, the most appropriate and realistic evaluation could be done under in-vivo conditions. While in-vivo tests are limited by several drawbacks, such as a pure control on desirable parameters, individual variance among subjects and requirements for a large and homogeneous subject group.

Therefore, the purpose of this present study was to develop in-vivo method for manipulation of skin oxygen saturation, and evaluate this approach by means of hyperspectral imaging assessing cutaneous blood oxygen saturation.

*zbigis@latnet.lv; phone +371 67033920

\section{METHODS}

\subsection{Experimental procedure}

The present study was conducted in accordance with The Helsinki Declaration and approved by Ethics Committee of the University of Latvia. Prior to the study, every subject was informed about all procedures, particularly emphasizing on the possible discomfort during long occlusion and of blood sampling procedure from the fingers. Thirteen healthy subjects ( $25 \pm 5$ y.o., mean $\pm \mathrm{SD}$ ) gave informed consent and were enrolled in the study.

To insure adequate microcirculation, the surface temperature of the right middle and index fingers was measured with a contactless thermometer (Raytech Inc.) and subjects with the skin temperature below $27^{\circ} \mathrm{C}$, were excluded from the study because of reduced cutaneous blood flow and possibly compromised baseline skin saturation.

Before experimental procedure, subject was seated in a comfortable chair with supported hands. The entire protocol comprises four consecutive stages, see Fig.1.A. During the first stage, an intact finger (second index finger) was disinfected with isopropyl alcohol pad and pricked using finger picker (soft click, Accucheck). The small capillary blood sample $(120 \mathrm{uL})$ was collected using heparinized sampling tube (Accriva Diagnostics) and transferred to CG4+ cartridge for analysis with a portable whole blood analyzer (iSTAT, Abbott).
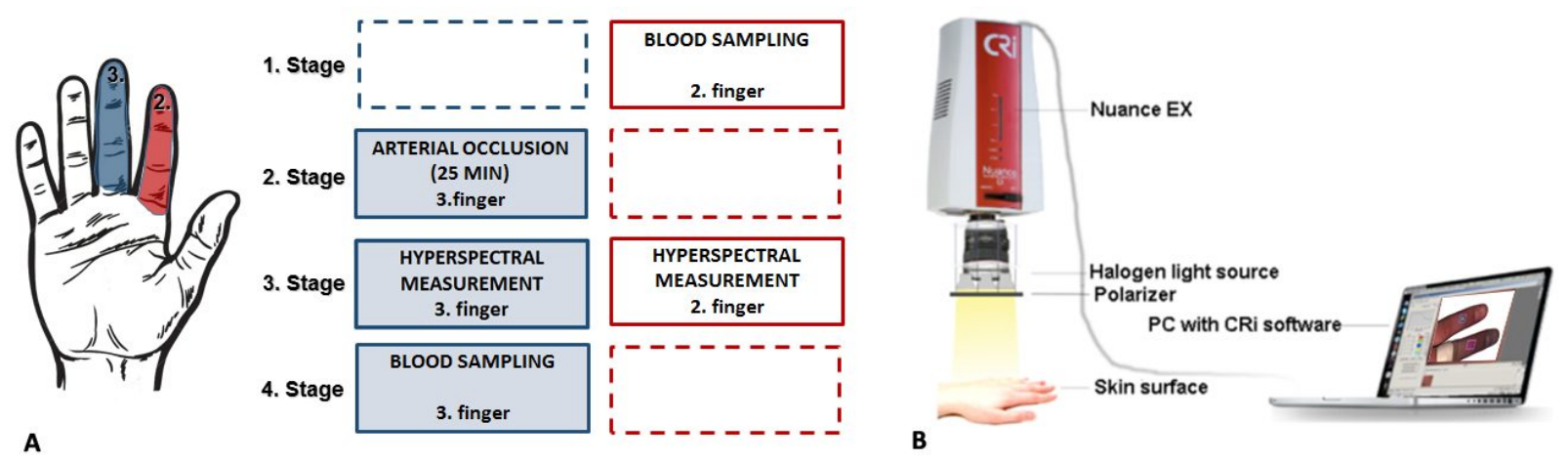

Figure 1. Finger arterial occlusion protocol (A) and acquisition of skin hyperspectral cube (B). Blue color indicates occluded finger (3), red color- intact finger (2). 
In order to produce alterations of skin capillary blood biochemical parameters and oxygen saturation, in the second stage of protocol the proximal phalanx of the right middle finger was occluded with a pneumatic cuff at the $150 \mathrm{mmHg}$ pressure and kept occluded for 25 minutes.

Seemingly long finger arterial occlusion time is common in clinics and laboratory settings and not harmful to subjects. Performed as a routine during orthopedic operations; with the occlusion time around $2 \mathrm{~h}$ and the cuff pressure $50 \mathrm{mmHg}$ above patient systolic blood pressure. The recommended single occlusion time should not exceed 30 minutes for elderly and critically ill patients ${ }^{5}$, following the 3-5 min post-occlusion period prior to next occlusion ${ }^{6}$.

There are several papers describing normal physiological studies (healthy volunteers) with the limb occlusion up to 30 minutes at suprasystolic cuff pressure without any harm to subjects ${ }^{7-10}$. Moreover, in our study, subjects were able to interrupt occlusion procedure at any moment.

In the third stage of protocol during the last minute $\left(25^{\text {th }}\right)$ of occlusion, the hyperspectral cube (HIS cube) from the hairy skin of both occluded (middle) and intact (index) finger was captured at $450 \mathrm{~nm}-800 \mathrm{~nm}$ range and $5 \mathrm{~nm}$ spectral resolution with tunable filter hyperspectral camera, Nuance EX (CRi, UK) as depicted in Fig.1.B. The HIS cube acquisition procedure has been described in detail elsewhere ${ }^{11}$.

Within the minute after hyperspectral acquisition, capillary blood was collected from the occluded finger and analyzed by the same portable analyzer- fourth stage. After that, pneumatic cuff has been released and the finger returned to the normal state within the following 1-3 minutes.

The six tissue ischemia characterizing blood parameters were obtained from whole blood analyzer from intact and occluded finger: $\mathrm{pCO}_{2}$ - carbon dioxide partial pressure, $\mathrm{pO}_{2}$ - oxygen partial pressure, Lact- lactate concentration, $\mathrm{pH}$ acidity, $\mathrm{HCO}^{3}$ - bicarbonate concentration and $\mathrm{SaO}_{2}-\mathrm{Hemoglobin}$ saturation with the oxygen.

\subsection{The processing of hyperspectral data}

Oxygen saturation was calculated using light diffusion model in the $\operatorname{skin}^{12,13}$.

In the modeling process it has been assumed that skin consists of three layers: stratum corneum (SC), epidermis (epid.), and dermis (derm.). Another assumption was that SC does not absorb light, only providing scattering of the light as it contains mainly keratin. Epidermal layer has skin pigment melanin which absorbs light and the main absorber in the dermal layer is blood, which can be divided in absorption of oxy/deoxy-hemoglobin $\left(\mathrm{HbO}_{2}\right.$ and $\left.\mathrm{HbD}\right)$.

Accordingly, absorption coefficients are:

$$
\mu_{\text {a_epid }}=C_{m e l} \cdot \varepsilon_{m e l}, \quad \mu_{a_{-} \text {derm }}=C_{h b O 2} \cdot \varepsilon_{h b O 2}+C_{h b D} \cdot \varepsilon_{h b D,}
$$

where $C_{m e l}, C_{h b O 2}, C_{h b D}$ is chromophore concentration in skin, $\varepsilon_{m e l}, \varepsilon_{h b O 2}, \varepsilon_{h b D}$ is extinction coefficients of melanin, $\mathrm{HbO}_{2}$ and $H b D$.

The scattering occurs in whole skin, each layer has different scattering properties. The model is sophisticated as the scattering depends also on absorption in skin chromophores ${ }^{14}$ :

$$
\mu_{s}=\mu_{s_{-} 500} \cdot\left[\rho \cdot(\lambda / 500)^{-4}+(1-\rho) \cdot(\lambda / 500)^{-\gamma}\right],
$$

where $\mu_{s 500}$ is reference scattering at $\lambda=500 \mathrm{~nm}, \rho, \gamma$-scattering parameters. Attenuation coefficient is $\mu=\mu_{a}+\mu_{s}$.

Using 3-layer light diffusion model, we obtain reflectance spectrum $R(\lambda)$ of the model.

Reflectance depends on $C_{m e l}, C_{h b O 2}, C_{h b D}$ and model parameters $\mu_{s 500 s c}, \mu_{s 500 e p i d}, \rho_{s c}, \rho_{\text {epid }}, \gamma_{s c}, \gamma_{\text {epid }}$ and layers thicknesses. Chromophore concentrations are found fitting reflectance spectrum of the model to measured reflectance. Since model parameters can slightly deviate from expected [3], we allow change in these parameters within a small range using fitting.

The final skin microcirculation parameter is oxygen saturation $\left(\mathrm{SaO}_{2}\right)$, which can be found from the following equation:

$$
\mathrm{SaO}_{2}=100 \cdot C_{h b O 2} /\left(C_{h b O 2}+C_{h b D}\right) \text {. }
$$


For off-line analysis of HSI data acquired by Nuance camera, image processing software was developed in the Matlab GUI environment (Fig. 2). This software allows visualization of oxygen saturation maps, mapping its distribution in different skin regions of different body parts. Computation of maps is performed by algorithmic steps described in our previous study ${ }^{11}$.

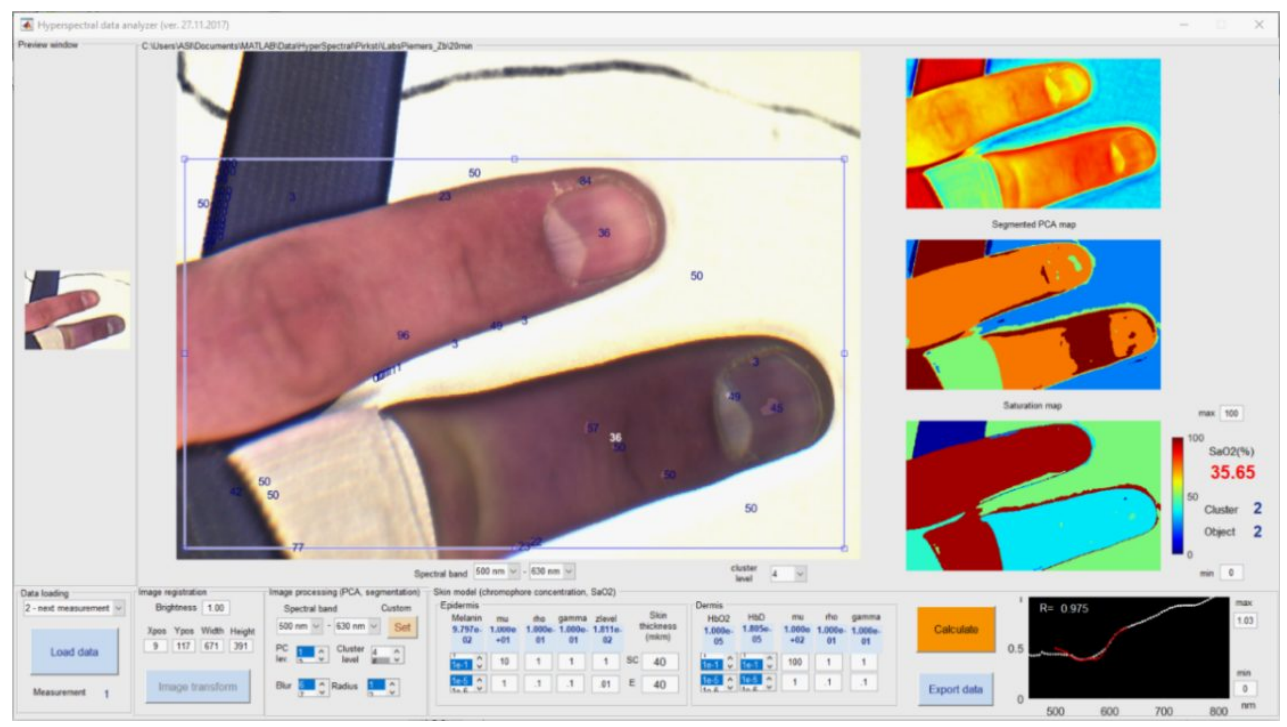

Figure 2. The screenshot of software interface for hyperspectral data analyses and blood saturation mapping. The fingers photo was digitally contrasted to emphasize skin color tone diferences.

\subsection{Statistical analyses}

The statistical analysis was performed using (SigmaPlot12, Systat Software) statistical software. The group values were expressed as the arithmetic mean \pm standard deviation. To compare the group mean values, Student-t test was utilized, considering $\mathrm{p}<0.005$ significance level. The comparison of methods (HIS vs REF blood analyzer) was performed utilizing Bland-Altman Analyses.

\section{RESULTS AND DISCUSSION}

\subsection{Capillary blood biochemical parameters}

The baseline values of capillary blood parameters in the intact finger were very similar for all subjects, displaying small standard deviation values, arithmetic mean \pm standard deviation values are as follow: $\mathrm{pCO}_{2}-36.77 \pm 3.95 \mathrm{mmHg}, \mathrm{pO}_{2}$ $74.20 \pm 5.97 \mathrm{mmHg}$, Lact-1.72 $\pm 0.85 \mathrm{mmol} / \mathrm{L}, \mathrm{pH}-7.44 \pm 0.03, \mathrm{HCO}^{3}-24.93 \pm 2.07 \mathrm{mmol} / \mathrm{L}$ and $\mathrm{SaO}_{2}-95.13 \pm 1.46 \%$.

The obtained values were slightly lower than typical arterial values indicating normal metabolism, cardiopulmonary function and optimal cutaneous blood supply ${ }^{1516}$. The obtained capillary blood hemoglobin saturation values were in the physiological range, hence slightly lowered in comparison to arterial blood.

Taking into the consideration sufficient cutaneous perfusion, as confirmed by skin temperature measurement, and relatively small volume of fingers, the values of these blood parameters can be attributed to the cutaneous circulation, which is partly determined by superficial vascular plexus situated in papillary dermis.

Similar baseline values for oxygen and carbon dioxide concentrations have been reported during transcutaneous gas measurement: $\mathrm{tcO}_{2}=73.9 \pm 14.6 \mathrm{mmHg}$ and $\mathrm{tcCO}_{2}=40.5 \pm 9.8 \mathrm{mmHg}$ supporting our findings ${ }^{17}$. Our assumption is 
advocated by the observation of local differences between capillary blood values from different sites of the body ${ }^{18}$. The different tissue has different energetic metabolism, perfusion and capillary density ${ }^{19}$. The hands and fingers are unique as they have very high capillary density and majority of capillaries are situated in the skin papillary dermis (approx. 7 times difference in density to other body sites), close to the skin surface ${ }^{19}$. The dermis exhibits an extensive vasculature that is arranged in two layers that are parallel to the skin surface. The superficial plexus is situated between the papillary and the upper reticular dermis, while deep plexus in the lower reticular dermis, and are connected by perpendicularly orientated communicating vessels, the capillary loop rise upwards into the papillae from the subpapillary plexus ${ }^{20}$. The major determinant of cutaneous oxygen consumption is metabolism, which occurs in all layers of epidermis and dermis except stratum corneum. The oxygen demand is partially covered by the capillary blood supply and partly by diffusion from the atmosphere through the skin's upper surface. In contrast, the epidermis has no vasculature, but is exposed directly to the atmosphere ${ }^{4}$.
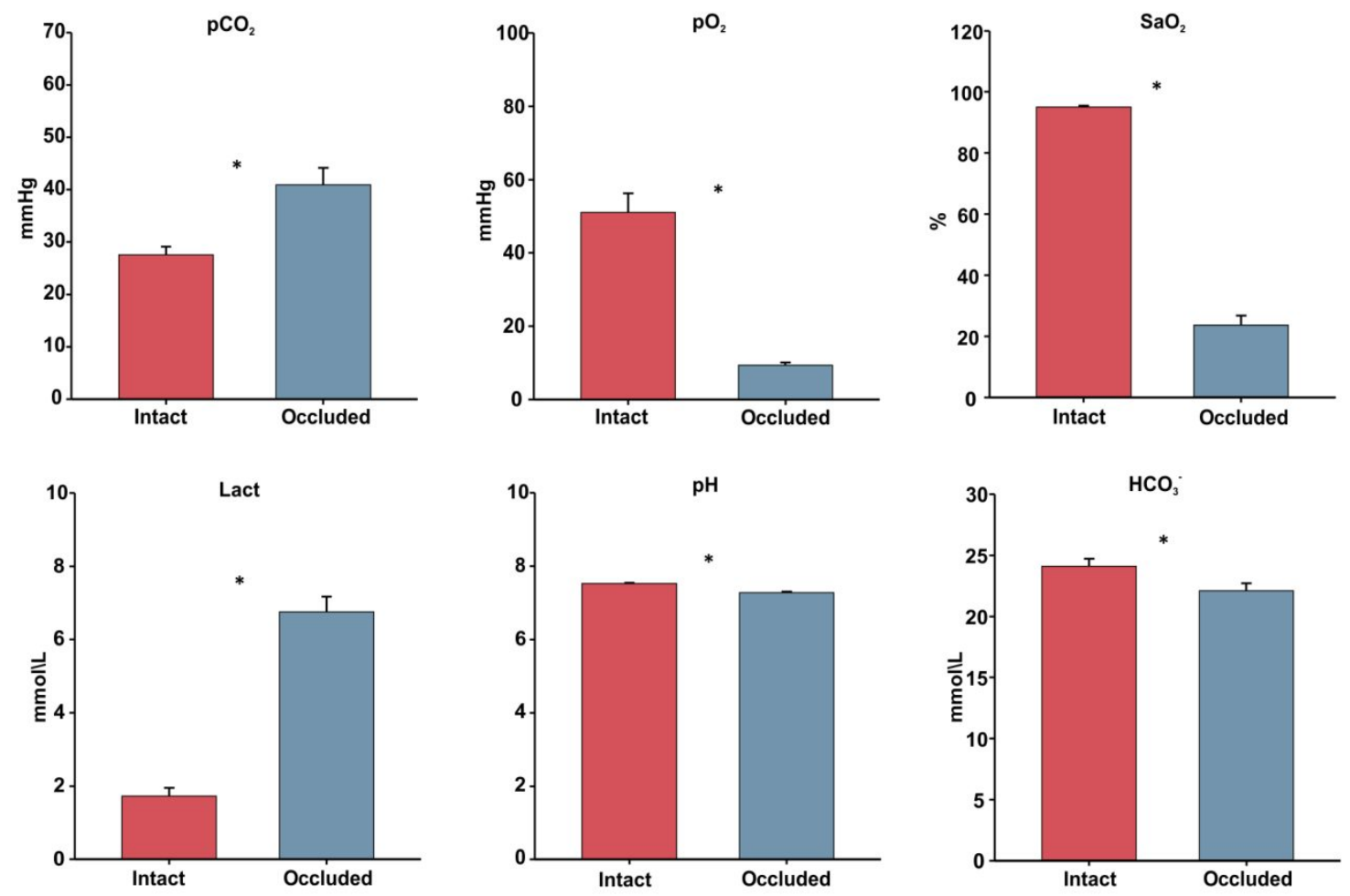

Figure 3. Changes of capillary blood parameters during baseline (in intact finger) and occlusion (in occluded finger), reported values are group mean \pm standard deviation, statistically significant difference $(\mathrm{p}<0.05)$ is denoted with asterisk

Our approach on alteration of skin blood saturation is based on induction of hypoxia to the skin and underlying tissue by arresting blood supply, which should substantially change capillary blood parameters in occluded tissue. As expected earlier, twenty five minutes of finger arterial occlusion produced significant alterations of blood parameters proximally to occlusion site in all subjects, resembling blood gas profile seen in the clinics during severe acidosis and hypoxemia ${ }^{1521}$. The mechanism of occlusion is well known, as occlusion is utilized as a routine procedure during orthopedic surgery ${ }^{22-24}$. During arrested arterial blood supply and venous blood drainage, occluded tissue try to maintain metabolism resulting in utilization of blood oxygen and production of carbon dioxide. After diminishing oxygen reserves, tissue energetic metabolism switches to anaerobic, substantially increasing acidity and concentration of anaerobic byproducts. Finger occlusion produced significant alterations of capillary blood for all 13 subjects indicating acidosis and hypoxemia in the occluded region. The observed blood values were as follow: $\mathrm{pCO}_{2}-73.83 \pm 13.40 \mathrm{mmHg}$, $\mathrm{pO}_{2}-21.93 \pm 4.62 \mathrm{mmHg}$, Lact-6.60 $\pm 1.22 \mathrm{mmol} / \mathrm{L}, \mathrm{pH}-7.24 \pm 0.09, \mathrm{HCO}^{3}-23.20 \pm 2.28 \mathrm{mmol} / \mathrm{L}$ and $\mathrm{SaO}_{2}-22.73 \pm 9.09 \%$, demonstrating larger dispersion of blood values in comparison to the baseline, possible because of different metabolic 
rate and size of the occluded tissue. Similar data has been obtained in occlusion in other studies, confirming our results ${ }^{24}$. The comparison of group mean values in intact and occluded finger capillary blood parameters are depicted in Figure 3.

The observed capillary blood changes in occluded region - occluded finger last for less than a minute, following release of occlusion cuff, without any harm to the subjects. Because of relatively short occlusion time, small occluded tissue volume and relatively high capacity of peripheral tissue (finger) to adapt for hypoxia ${ }^{25}$. The significant blood hemoglobin saturation drop has been achieved in the occluded finger as expected. Hence, occlusion influenced other undesirable blood parameters such as concentration of lactate, partial pressure of carbon dioxide and $\mathrm{pH}$, which is considered as the side effect in our finger occlusion model, cannot be eliminated, due to the nature of physiological regulation mechanisms.

\subsection{Skin hyperspectral parameters}

During first minutes of occlusion, subjects felt slight discomfort which almost disappeared after 4-7 minutes following application of occlusion cuff and at the end of occlusion, the finger had reduced sensation. During the time course of occlusion, the restrained finger became darker, displaying signs of mild cyanosis. For some subjects was remarkable, due
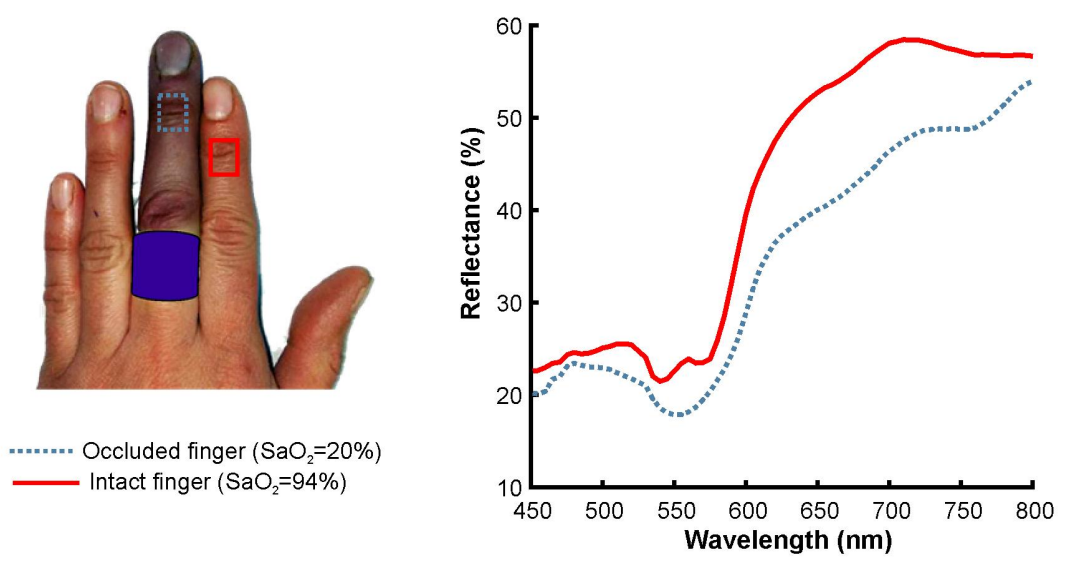

Figure 4. Skin spectra and photo of intact (solid line) and occluded (dotted line) tissue at baseline, in intact finger and occlusion, in occluded finger. The photo of hand was digitally contrasted (25\%) to show skin color tones of intact and occluded finger.

to the blood pooling in superficial vascular plexus situated in papillary dermis. It has been supported by our observation that mechanical compression of skin tissue produced white patches, as the capillary blood pools were dispersed. The typical cyanotic finger is depicted in Figure 4 on the left side, with the corresponding spectrum acquired from intact and occluded skin, pointing on the obvious difference in hemoglobin maximal absorption spectral region 500-600nm.
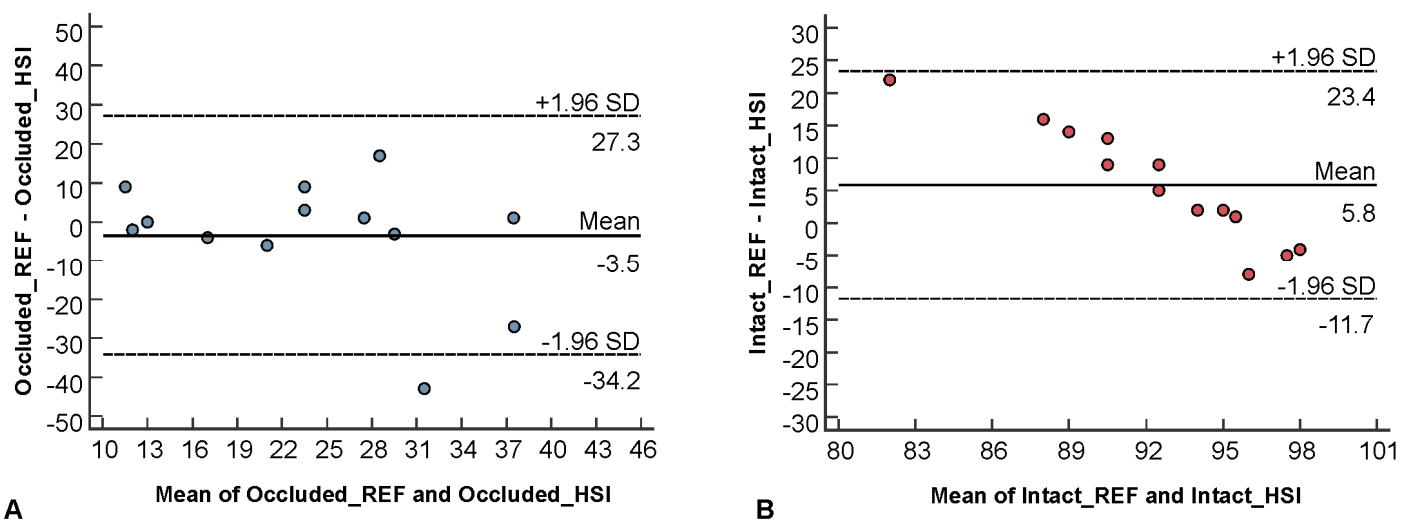

Figure 5. Bland-Altman plot of agreement between saturation obtained by referent (REF) and hyperspectral method (HIS). From occluded finger during $25^{\text {th }}$ minute of occlusion (A) and intact finger during baseline (B). 
In order to hyperspectrally evaluate the reliability of finger occlusion model, an assumption that oxygen is distributed uniformly in occluded tissue region has been made, which is the matter of discussion and controversies in the literature.

The mean baseline value for intact skin saturation acquired by the blood analyzer was $95.13 \pm 1.46 \%$, which resembles arterial blood. Controversial data have been reported in the literature regarding baseline values of skin capillary blood saturation which were recorded with hyperspectral imaging- ranging from $50 \%-96 \%{ }^{26-31}$.

The diffusion of gases within relatively small tissue volume depends on many factors such as tissue temperature, gas concentration gradient and tissue mechanical composition, and within 25 minutes should lead to equal distribution of oxygen in skin and underlying soft tissue. Therefore it has been assumed that skin saturation values are represented by saturation of capillary blood taken from the skin microvasculature. The group mean value for $\mathrm{HIS}$ derived $\mathrm{SaO}_{2}$ in intact finger skin was $89.46 \pm 8.79 \%$, while substantially decreasing in occluded finger: $25.85 \pm 14.00 \%$, displaying small difference between two independent techniques, as seen in Figure 5.

The HIS derived mean saturation value display substantially higher dispersion compared to the referent saturation (standard deviations) indicated on possible differences of skin structure or capillary density across the subjects as the tissue model parameters remained the same.

\section{CONCLUSIONS}

The obtained skin blood oxygen saturation values were similar for the whole blood analyzer and hyperspectral imaging technique, indicating the reliability of our proposed finger arterial occlusion model. However, further studies are required to improve reproducibility of occlusion induced saturation changes and quantify skin thickness to improve accuracy of utilized tissue diffusion model.

\section{ACKNOWLEDGEMENTS}

This work was supported by the European Regional Development Fund project \#1.1.1.1/16/A/065 "Optical Non-invasive Hybrid Method for Early Diagnostics and Therapy Guidance in Sepsis".

\section{REFERENCES}

[1] Wukitsch, M. W., "Pulse oximetry: historical review and Ohmeda functional analysis.," Int. J. Clin. Monit. Comput. 4(3), 161-166 (1987).

[2] Schmidt, S., Langner, K., Dudenhausen, J. W. and Saling, E., "Reliability of transcutaneous measurement of oxygen and carbon dioxide partial pressure with a combined Po2-Pco2 electrochemical sensor in the fetus during labor.," J. Perinat. Med. 13(3), 127-133 (1985).

[3] Diebele, I., Kuzmina, I., Kapostinsh, J., Derjabo, A. and Spigulis, J., "Melanoma-nevus differentiation by multispectral imaging,” Proc. SPIE-OSA Biomed. Opt., N. Ramanujam and J. Popp, Eds., 80872G-80872G-6 (2011).

[4] Lenasi, H., "Assessment of Human Skin Microcirculation and Its Endothelial Function Using Laser Doppler Flowmetry," [Medical Imaging], F. Okechukwu Erondu, Ed., InTech, 412 (2011).

[5] Horlocker, T. T., Hebl, J. R., Gali, B., Jankowski, C. J., Burkle, C. M., Berry, D. J., Zepeda, F. A., Stevens, S. R. and Schroeder, D. R., "Anesthetic, patient, and surgical risk factors for neurologic complications after prolonged total tourniquet time during total knee arthroplasty.," Anesth. Analg. 102(3), 950-955 (2006).

[6] Sharma, J. P. and Salhotra, R., "Tourniquets in orthopedic surgery.," Indian J. Orthop. 46(4), 377-383 (2012).

[7] Thijssen, D. H. J., Bleeker, M. W. P., Smits, P. and Hopman, M. T. E., "Reproducibility of blood flow and postocclusive reactive hyperaemia as measured by venous occlusion plethysmography," Clin. Sci. (Lond). 108(2), 151-157 (2005).

[8] Ridout, S. J., Parker, B. A. and Proctor, D. N., "Age and regional specificity of peak limb vascular conductance in women.," J. Appl. Physiol. 99(6), 2067-2074 (2005).

[9] Thijssen, D. H. J., Kooijman, M., de Groot, P. C. E., Bleeker, M. W. P., Smits, P., Green, D. J. and Hopman, M. T. E., "Endothelium-dependent and -independent vasodilation of the superficial femoral artery in spinal cord- 
injured subjects.," J. Appl. Physiol. 104(5), 1387-1393 (2008).

[10] Pump, B., Schou, M., Gabrielsen, A. and Norsk, P., "Contribution of the leg vasculature to hypotensive effects of an antiorthostatic posture change in humans.," J. Physiol. 519 Pt 2, 623-628 (1999).

[11] Saknite, I., Grabovskis, A., Kazune, S., Rubins, U., Marcinkevics, Z., Volceka, K., Kviesis-Kipge, E. and Spigulis, J., "Novel hybrid technology for early diagnostics of sepsis," Proc. SPIE 10057, 100570F (2017).

[12] Spott, T., Svaasand, L. O., Anderson, R. E. and Schmedling, P. F., "Application of optical diffusion theory to transcutaneous bilirubinometry,” Proc. SPIE 3195, 234-245 (1998).

[13] Svaasand, L. O., Norvang, L. T., Fiskerstrand, E. J., Stopps, E. K. S., Berns, M. W. and Nelson, J. S., "Tissue parameters determining the visual appearance of normal skin and port-wine stains," Lasers Med. Sci. 10(1), 5565 (1995).

[14] Iglesias-Guitian, J. A., Aliaga, C., Jarabo, A. and Gutierrez, D., "A Biophysically-Based Model of the Optical Properties of Skin Aging," Comput. Graph. Forum 34(2), 45-55 (2015).

[15] Larkin, B. G. and Zimmanck, R. J., "Interpreting Arterial Blood Gases Successfully," AORN J. 102(4), 343-357 (2015).

[16] Phypers, B. and Pierce, J. T., "Lactate physiology in health and disease," Contin. Educ. Anaesth. Crit. Care Pain 6(3), 128-132 (2006).

[17] Rodrigues, L. M., Pinto, P. C. and Leal, A., "Transcutaneous flow related variables measured in vivo: the effects of gender," BMC Dermatol. 1(1), 4 (2001).

[18] Zavorsky, G. S., Cao, J., Mayo, N. E., Gabbay, R. and Murias, J. M., “Arterial versus capillary blood gases: a meta-analysis.," Respir. Physiol. Neurobiol. 155(3), 268-279 (2007).

[19] Pasyk, K. A., Thomas, S. V, Hassett, C. A., Cherry, G. W. and Faller, R., "Regional differences in capillary density of the normal human dermis.," Plast. Reconstr. Surg. 83(6), 939-45-7 (1989).

[20] Braverman, I. M., "Ultrastructure and organization of the cutaneous microvasculature in normal and pathologic states.," J. Invest. Dermatol. 93(2 Suppl), 2S-9S (1989).

[21] Sood, P., Paul, G. and Puri, S., "Interpretation of arterial blood gas.," Indian J. Crit. Care Med. 14(2), 57-64 (2010).

[22] Huang, Z., Ma, J., Zhu, Y., Pei, F., Yang, J., Zhou, Z., Kang, P. and Shen, B., "Timing of Tourniquet Release in Total Knee Arthroplasty," Orthopedics 38(7), 445-451 (2015).

[23] Girardis, M., Milesi, S., Donato, S., Raffaelli, M., Spasiano, A., Antonutto, G., Pasqualucci, A. and Pasetto, A., "The hemodynamic and metabolic effects of tourniquet application during knee surgery.," Anesth. Analg. 91(3), 727-731 (2000).

[24] Kang, H. J., Han, C. D., Jahng, J. S. and Ko, S. O., "Blood gas and electrolyte changes after tourniquet application in total knee replacement surgery," Yonsei Med. J. 33(2), 153 (1992).

[25] Michiels, C., "Physiological and pathological responses to hypoxia.," Am. J. Pathol. 164(6), 1875-1882 (2004).

[26] Lundsgaard, C. and Möller, E., "Investigation of the oxygen content of cutaneous blood (So called capillary blood)," J. Exp. Med. 36(5), 559-573 (1922).

[27] Chin, M. S., Freniere, B. B., Lo, Y.-C., Saleeby, J. H., Baker, S. P., Strom, H. M., Ignotz, R. a., Lalikos, J. F. and Fitzgerald, T. J., "Hyperspectral imaging for early detection of oxygenation and perfusion changes in irradiated skin," J. Biomed. Opt. 17(2), 26010 (2012).

[28] Huang, J., "Multispectral Imaging of Skin Oxygenation" (2012).

[29] Jafari-saraf, L., Wilson, S. E. and Gordon, I. L., "Hyperspectral Image Measurements of Skin Hemoglobin Compared With Transcutaneous PO 2 Measurements,” Ann. Vasc. Surg. 26(4), 537-548 (2012).

[30] Vyas, S., Banerjee, A. and Burlina, P., "Estimating physiological skin parameters from hyperspectral signatures.," J. Biomed. Opt. 18(5), 57008 (2013).

[31] Yudovsky, D. and Pilon, L., "Retrieving skin properties from in vivo spectral reflectance measurements," J. Biophotonics 4(5), 305-314 (2011). 